\title{
Duodenal mucinous adenocarcinoma presenting as ileus, obstructive jaundice and massive ascites: A case report
}

\author{
DONGBING DING $^{1}$, YAO YAO ${ }^{2}$ and SONGBAI ZHANG ${ }^{1}$ \\ Departments of ${ }^{1}$ Gastrointestinal Surgery and ${ }^{2}$ Ophthalmology, \\ Jingmen First People's Hospital, Jingmen, Hubei 448000, P.R. China
}

Received March 25, 2017; Accepted October 25, 2017

DOI: $10.3892 / \mathrm{mco} .2018 .1552$

\begin{abstract}
Duodenal mucinous adenocarcinoma (DMA) is a malignancy with a rather low morbidity. However, its incidence in China has not been clearly determined. We herein report a case of a 70-year-old female patient who presented with ileus, obstructive jaundice and massive ascites simultaneously. Gastroendoscopy revealed copious amounts of colloidal mucin in the gastric cavity. The neoplasm was not visualized on computed tomography or magnetic resonance imaging, and an exploratory laparotomy was performed to investigate the abdomen. During the operation, the anterior wall of the gastric antrum was incised to explore the first and second portions of the duodenum, as well as the gastric lumen, and a neoplasm sized $\sim 2.0 \mathrm{~cm}$ was identified at the antimesenteric border of the duodenal bulb after clearing the colloidal mucin content of the stomach and duodenum. Gastrojejunostomy rather than radical resection was performed due to the poor condition of the patient. The postoperative histopathological examination of the colloidal substance removed from the duodenal cavity revealed mucinous adenocarcinoma. On immunohistochemical analysis, the tissue stained positive for pan-cytokeratin, cytokeratin 19, CDX-2, carcinoembryonic antigen and Ki-67 $(8 \%)$, and negative for excision repair-1. The general condition of the patient postoperatively was poor; thus, adjuvant chemotherapy was not administered and the patient finally succumbed to the disease 42 days after surgery.
\end{abstract}

\section{Introduction}

Primary duodenal cancer (PDC) is rare, accounting for only a small proportion of gastrointestinal malignancies. The most frequent symptoms of PDC include abdominal pain, vomiting and weight loss. However, the clinical presentations of PDC are

Correspondence to: Dr Yao Yao, Department of Ophthalmology, Jingmen First People's Hospital, 67 Xiangshan Avenue, Jingmen, Hubei 448000, P.R. China

E-mail: yaoyaoddb@aliyun.cn

Key words: mucinous adenocarcinoma, duodenum, ileus, obstructive jaundice, ascites non-specific. The most common type of PDC is adenocarcinoma and it frequently occurs in the descending duodenum (1). Mucinous adenocarcinoma (MA) is derived from the epithelium, is characterized by the production of copious amounts of mucin, and is considered as a distinct pathological entity with poor prognosis. MA has been described in the breast, ovary, vulva, lung, pancreas, stomach, appendix and colorectum, but rarely in the duodenum $(2,3)$. As duodenal mucinous adenocarcinoma (DMA) is rare, its typical clinical presentation has not been well-documented. Gastroduodenoscopy and gastrointestinal barium radiography are considered as effective auxiliary examinations in the diagnosis of DMA. Currently, surgical resection is the standard treatment of choice for DMA, and the patients may benefit markedly from curative surgery with negative resection margins (1).

To the best of our knowledge, this is the first report of DMA presenting as ileus, obstructive jaundice and massive ascites simultaneously.

\section{Case report}

A 70-year-old female patient was admitted to the First People's Hospital of Jingmen (Jingmen, China) in February 2016 due to gradually aggravated abdominal pain and distension accompanied by jaundice for 10 days. The pain was localized to the subxiphoid and right upper abdominal areas, with paroxysmal exacerbations. There was no associated fever, nausea or vomiting. The abdominal examination revealed positive shifting dullness and prominent direct tenderness in the subxiphoid area and the right upper quadrant, with no rebound tenderness or muscle spasm. The bowel sounds were diminished. No subcutaneous varicose veins on the abdominal wall or gastrointestinal peristaltic wave was observed. The patient had undergone cholecystectomy and choledochoduodenostomy 10 years prior, but further information was unavailable. Hematological investigations revealed an elevated white blood cell count of $13.5 \times 10^{9} / 1$ (normal range, 3.5-9.5 $\times 10^{9} / 1$ ), a hemoglobin level of $85 \mathrm{~g} / 1$ (normal range, 115-150 g/l) and a platelet count of $164 \times 10^{9} / 1$ (normal range, 100-300x10 $/ 1$ ). Hepatic function tests revealed a total bilirubin concentration of $127.8 \mu \mathrm{mol} / 1$ (normal range, $0-21 \mu \mathrm{mol} / \mathrm{l}$ ), a direct bilirubin concentration of $112.2 \mu \mathrm{mol} / 1$ (normal range, $0-7 \mu \mathrm{mol} / \mathrm{l}$ ), an albumin concentration of $23.8 \mathrm{~g} / 1$ (normal range, 40-55 g/l), an alkaline phosphatase concentration of $410.0 \mathrm{U} / 1$ (normal 
range, $35-125 \mu \mathrm{mol} / \mathrm{l})$, and a $\gamma$-glutamate transpeptidase concentration of $135.0 \mathrm{U} / 1$ (normal range, 4-60 $\mu \mathrm{mol} / \mathrm{l}$ ). There was renal insufficiency, with elevated creatinine concentration (140.9 $\mu \mathrm{mol} / 1$; normal range, $41-84 \mu \mathrm{mol} / \mathrm{l})$. The level of the C-reactive protein was $148.5 \mathrm{mg} / 1$ (normal range, $0-10 \mathrm{mg} / \mathrm{l}$ ). The levels of carbohydrate antigen (CA) 125 and CA199 were $418.0 \mathrm{U} / \mathrm{ml}$ (normal range, 0-35 U/ml) and $459.7 \mathrm{U} / \mathrm{ml}$ (normal range, 0-27 U/ml), respectively. The $\alpha$-fetoprotein (AFP), CA153 and carcinoembryonic antigen (CEA) levels were normal. Carcinoma cells were not found on exfoliative cytology of the ascitic fluid. An abdominal X-ray revealed an air-fluid level. Computed tomography (CT) scans revealed calculi and dilation of intrahepatic duct, choledochectasia, a possible choledochocyst and a large fluid collection in the abdominal and pelvic cavity; no duodenal neoplasm or enlarged lymph nodes were identified (Fig. 1). The results of magnetic resonance imaging (MRI) scans were similar to those of CT scans (Fig. 2). Due to the renal insufficiency, the patient did not undergo enhanced CT or MRI. A gastroscopy revealed a large amount of semi-transparent colloidal substance (Fig. 3). Symptomatic nutritional support therapy was conducted after hospitalization and the patient was actively investigated to determine the cause of the symptoms. However, abdominal pain and distension gradually increased and were accompanied by discontinuous nausea and vomiting. An etiology could not be identified based on the symptoms, signs and auxiliary examination. The possibility of a malignant neoplasm was considered. However, the CT and MRI scans did not reveal any lesions and cancer cells were not detected in exfoliative cytology examination of the ascitic fluid. Therefore, an exploratory laparotomy was conducted to investigate the status of the abdomen. Peritoneal cancer foci were not observed. The duodenum was obviously dilated, rather than a choledochocyst that was initially suspected based on the CT findigs (Fig. 4A). The gastric antrum was then incised to explore the duodenum and it was found to contain a copious amount of a colloidal substance (Fig. 5). After clearing this colloidal substance, a cancer lesion with an irregular margin, $2.0 \mathrm{~cm}$ in diameter, was identified in the antimesenteric border of the duodenal bulb (Fig. 4B), and DMA was considered as the possible diagnosis. Based on the findings in the abdomen, and since the patient was elderly and in poor general condition, a gastrojejunostomy rather than radical resection was performed. The postoperative histopathological examination of the colloidal tumor revealed MA (Fig. 6A and B). On immunohistochemical examination, the cancer tissue was positive for pan-cytokeratin, cytokeratin 19, CDX-2, CEA and Ki-67 (8\%), and negative for excision repair-1. The general condition of the patient postoperatively was poor and adjuvant chemotherapy was not administered. The patient succumbed to the disease 42 days after surgery.

Consent was obtained from the patient and her family regarding the publication of the case details and associated images.

\section{Discussion}

Primary DMA is an exceedingly rare type of duodenal cancer and little information on this subject is available in the literature. At present, the pathogenesis of DMA has not been fully

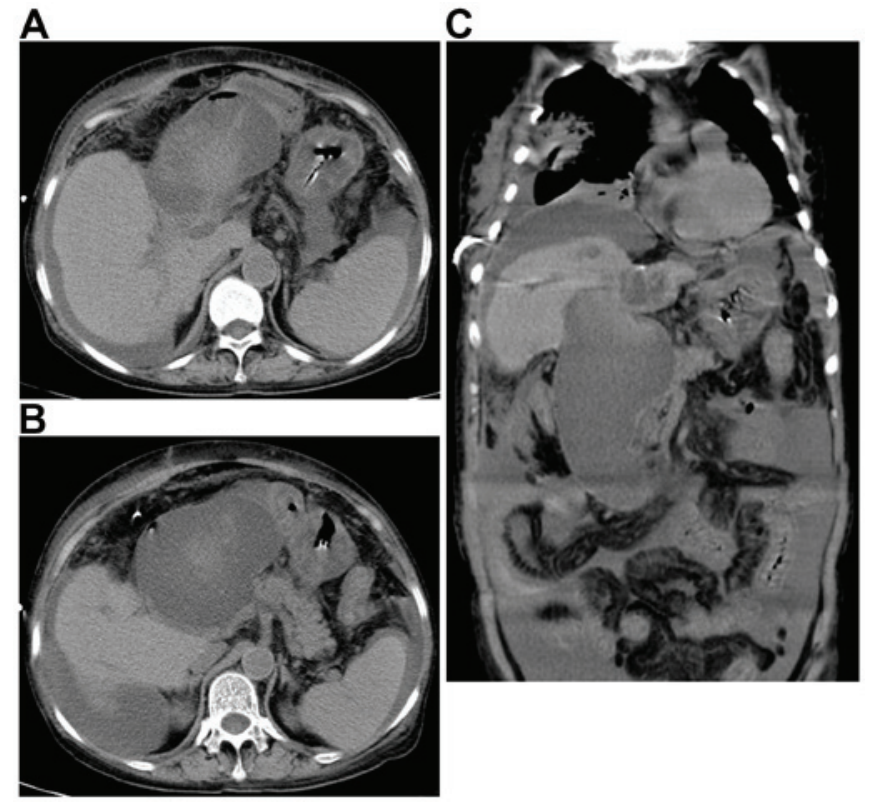

Figure 1. Computed tomography of the abdomen revealed dilation of the bulb and descending part of the duodenum, with massive ascites. No neoplasm was detected in the duodenum. (A and B) Transverse plane; (C) coronal plane.

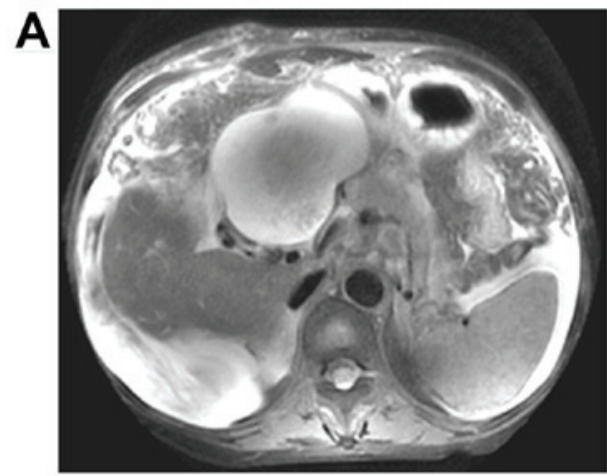

B



Figure 2. T2-weighted images on magnetic resonance imaging were similar to the computed tomography scans. (A) Transverse plane; (B) coronal plane.

elucidated. The low incidence rate and non-specific presentation increase the difficulty of DMA diagnosis.

Mucinous adenocarcinoma (MA) is diagnosed when $>50 \%$ of the neoplasm comprises mucinous cells on histological 


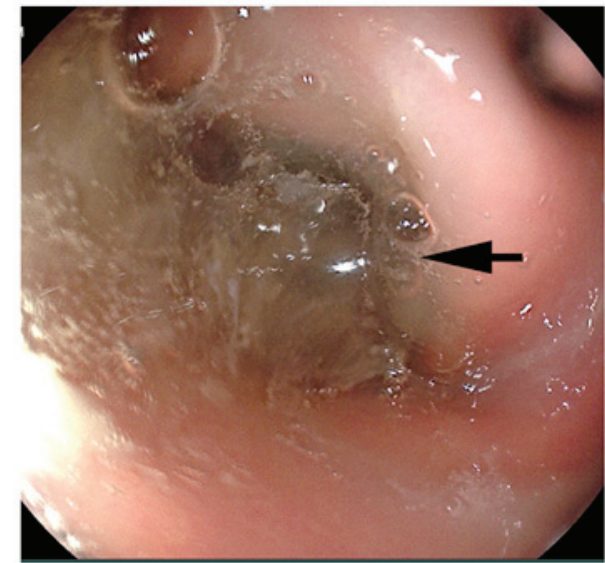

Figure 3. Esophagogastroduodenoscopy revealed copious amounts of mucin in the gastric lumen (arrow).
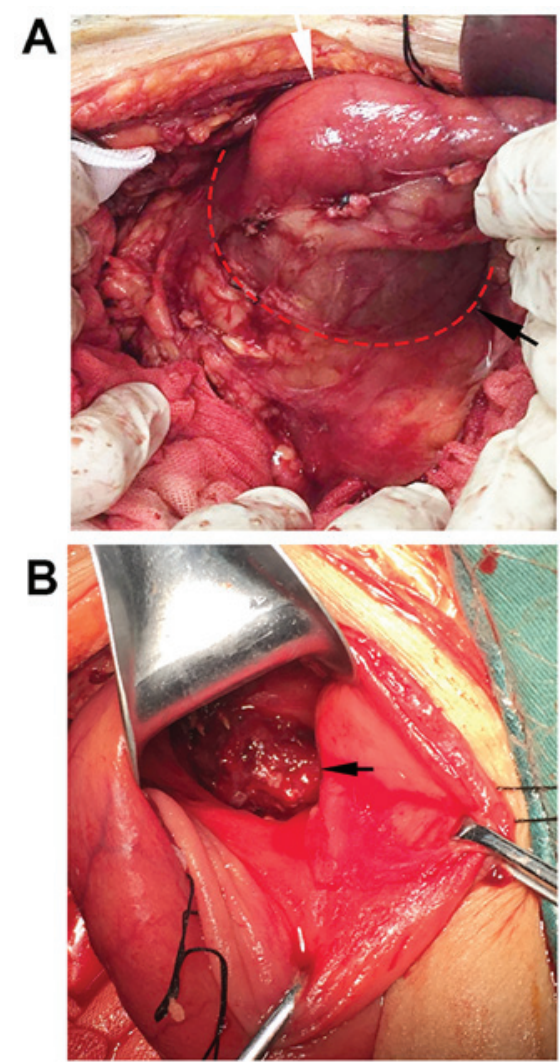

Figure 4. (A) The first and second portions of the duodenum were obviously dilated and slightly edematous (dotted line). White arrow, gastric antrum. Black arrow, dilated duodenum. (B) The anterior wall of the gastric antrum was incised to explore the first and second portions of the duodenum, and a neoplasm sized $\sim 2.0 \mathrm{~cm}$ (arrow) was identified in the antimesenteric border of the duodenal bulb after clearing the mucin.

examination, according to the criteria of the World Health Organization (4). MA may arise at various sites of the gastrointestinal tract, more frequently the stomach and colorectum, and the prognosis is unfavorable. Mucinous gastric carcinoma (MGC) is usually diagnosed at an advanced stage, and diagnosis of early MGC is extremely rare. Advanced-stage MGC is associated with a dismal prognosis, as its biological behavior is similar to that of other types of gastric cancer (5). Colorectal MA, a subtype of colorectal cancer, is associated with a high risk of

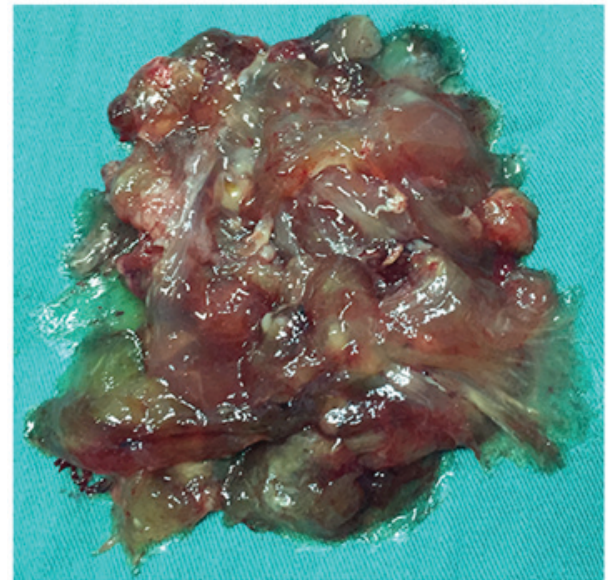

Figure 5. Colloidal substance removed from the gastric and duodenal lumen.
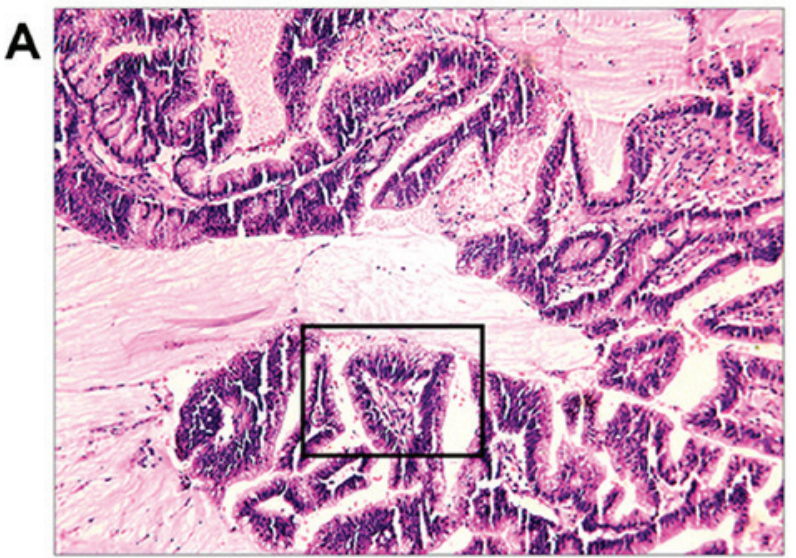

B

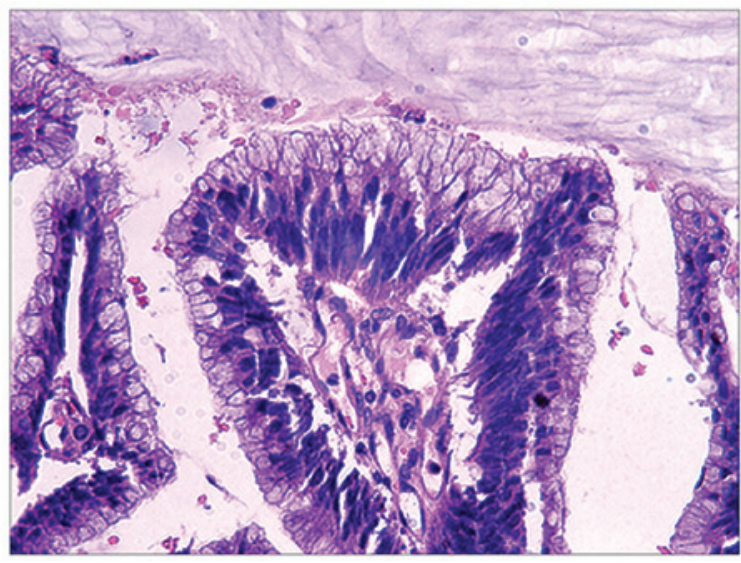

Figure 6. Histological examination of the colloidal substance removed from the duodenal cavity revealed mucinous adenocarcinoma. Hematoxylin and eosin staining; magnification, (A) x100 and (B) x400.

metastasis and a poorer prognosis compared with non-mucinous subtypes (6). The College of American Pathologists reported that the prognosis does not vary significantly among different MA subtypes, but rather relies on stage and grade (7). Generally, $\sim 0.1-1.3 \%$ of gastrointestinal malignancies are located in the small intestine, and the duodenum accounts for $>50 \%$ of those cases (8). Unfortunately, the majority of small bowel adenocarcinomas are diagnosed at an advanced stage, with metastasis in $35 \%$ of the cases (9). Thus, early diagnosis of DMA is crucial. 
The common symptoms of PDC include abdominal pain, vomiting, weight loss, gastrointestinal bleeding and jaundice (10).The cause of jaundice is frequently a peripapillary carcinoma, resulting in obstructive jaundice. In addition to abdominal pain, our patient presented with ileus, obstructive jaundice and massive ascites. The symptoms were attributed to the copious amount of colloidal substance accumulating in the duodenum. Gastroduodenoscopy and gastrointestinal barium radiography are commonly effective methods for diagnosing PDC (1). However, in the present case, only a large amount of colloidal substance was observed and the tumor in the duodenum was not identified. The presence of the colloidal substance was initially mistaken for a recently ingested food of similar consistency and the possibility of DMA was not considered. The role of $\mathrm{CT}$ in detecting PDC has not been adequately addressed. Zhang et al reported a sensitivity of $74.2 \%$ of CT scans in identifying the lesion in patients with PDC (1). Other studies reported that $\mathrm{CT}$ was effective for detecting cancerous lesions of the duodenum as well as for tumor staging preoperatively and postoperatively (11). However, there is currently no information on the DMA characteristics on CT and MRI. In the present case, CT and MRI scans revealed no signs of DMA. It may be hypothesized that the copious amount of the colloidal substance in the duodenum affected the findings on CT imaging. Gastroduodenoscopy is a valuable method for detection of duodenal cancers, and it has been reported that it may detect $\sim 90 \%$ of PDCs (12). Chae et al reported a DMA presenting as a laterally spreading tumor at the antimesenteric border of the second portion of the duodenum on upper gastroendoscopy, with no extracellular mucin or exudate due to the early stage (13). In the present case, the gastric cavity was filled with copious amounts of colloidal mucin on gastroendoscopy. However, the possibility of DMA was not considered at the time. Compared with early DMA, a large amount of mucin in the gastric cavity on gastroendoscopy may be a warning sign for advanced DMA. The levels of five serological tumor markers (CEA, CA199, CA125, CA724 and AFP) are valuable tools in the diagnosis, evaluation of prognosis and monitoring of the treatment response of several gastrointestinal cancers, although their role in predicting DMA has not been elucidated. Serum CA125 is a relatively sensitive tool for the differentiation of malignant ascites (14). In the present case, the serum CA125 level was markedly elevated and accompanied by massive ascites. The possibility of malignant ascites was considered, despite the uncertain location of the neoplasm. Therefore, increased serum tumor markers may be potential signals for DMA.

Since radical resection with a negative margin may prolong survival, curative surgery is considered as the best option for duodenal cancer. Chae et al reported the case of an asymptomatic patient with early DMA, and with the cancer only invading the submucosa; subsequently, Roux-en-Y duodenojejunostomy and jejunojejunostomy were performed (13), with no evidence of recurrence during 1 year of follow-up. Considering her poor condition, the patient in the present case was unable to tolerate complete resection; thus, a gastrojejunostomy was performed to relieve the obstruction. Unfortunately, the patient's general condition was poor postoperatively and adjuvant chemotherapy was not administered; she finally succumbed to the disease 42 days after surgery.
In conclusion, DMA is a rare malignancy with no specific manifestations. To the best of our knowledge, this is the first case of DMA presenting as ileus, obstructive jaundice and massive ascites simultaneously. Early detection and subsequent radical resection appear to be an effective way for improving the outcome. At present, preoperative diagnosis of DMA remains difficult and surgeons should include this neoplasm in the differential diagnosis when encountering the abovementioned symptoms. Further studies are required to establish standard protocols for the diagnosis and treatment of DMA.

\section{Competing interests}

The authors declare that they have no competing interests.

\section{References}

1. Zhang S, Cui Y, Zhong B, Xiao W, Gong X, Chao K and Chen M: Clinicopathological characteristics and survival analysis of primary duodenal cancers: A 14-year experience in a tertiary centre in South China. Int J Colorectal Dis 26: 219-226, 2011.

2. Sui Y, Zou J, Batchu N, Lv S, Sun C, DU J, Wang Q, Song Q and Li Q: Primary mucinous adenocarcinoma of the vulva: A case report and review of the literature. Mol Clin Oncol 4: 545-548, 2016.

3. Tan J, Yang S, Shen P, Sun H, Xiao J, Wang Y, Wu B, Ji F, Yan J, Xue $\mathrm{H}$ and Zhou D: C-kit signaling promotes proliferation and invasion of colorectal mucinous adenocarcinoma in a murine model. Oncotarget 6: 27037-27048, 2015.

4. Jass JR and Sobin LH: Histologic Typing of Intestinal Tumours 2nd edition. Berlin, Springer Verlag, 1989.

5. Yasuda K, Adachi Y, Shiraishi N, Yamaguchi K, Shiromizu A and Kitano S: Pathology and prognosis of mucinous gastric carcinoma. J Surg Oncol 76: 272-277, 2001.

6. Symonds DA and Vickery AL: Mucinous carcinoma of the colon and rectum. Cancer 37: 1891-1900, 1976.

7. Compton C, Fenoglio-Preiser CM, Pettigrew N and Fielding LP: American joint committee on cancer prognostic factors consensus conference: Colorectal working group. Cancer 88: 1739-1757, 2000.

8. Wilson JM, Melvin DB, Gray GF and Thorbjarnarson B: Primary malignancies of the small bowel: A report of 96 cases and review of the literature. Ann Surg 180: 175-179, 1974.

9. Halfdanarson TR, McWilliams RR, Donohue JH and Quevedo JF: A single-institution experience with 491 cases of small bowel adenocarcinoma. Am J Surg 199: 797-803, 2010.

10. Bakaeen FG, Murr MM, Sarr MG, Thompson GB, Farnell MB, Nagorney DM, Farley DR, van Heerden JA, Wiersema LM, Schleck CD and Donohue JH: What prognostic factors are important in duodenal adenocarcinoma? Arch Surg 135: 635-642, 2000.

11. Adedeji OA, Trescoli-Serrano C and Garcia-Zarco M: Primary duodenal carcinoma. Postgrad Med J 71: 354-358, 1995.

12. Han SL, Cheng J, Zhou HZ, Zeng QQ and Lan SH: The surgical treatment and outcome for primary duodenal adenocarcinoma. J Gastrointest Cancer 40: 33-37, 2009.

13. Chae MJ, Baek IH, Oh YM, Lim JU, Jeon JW, Shin HP, Joo KR and Lee JI: A Patient with duodenal mucinous adenocarcinoma presenting as a laterally spreading tumor. Clin Endosc 48: 336-339, 2015.

14. Trapé J, Gurt G, Franquesa J, Montesinos J, Arnau A, Sala M, Sant F, Casado E, Ordeig JM, Bergos C, et al: Diagnostic accuracy of tumor markers CYFRA21-1 and CA125 in the differential diagnosis of ascites. Anticancer Res 35: 5655-5660, 2015. 